\title{
LASER SPECTROSCOPIC INVESTIGATION OF HIGH RYDBERG STATES OF NO: DECAY DYNAMICS NEAR THE FIRST IONIZATION THRESHOLD
}

\author{
ASUKA FUJII and NORIO MORITA \\ Institute for Molecular Science, Myodaiji, Okazaki 444 Japan
}

(Received 15 March, 1993)

With a state-selective laser excitation, two main decay processes, autoionization and predissociation, in vibrational superexcited Rydberg states $(\mathrm{n}=8-12, l=\mathrm{s}, \mathrm{p}$, and $\mathrm{f}, \mathrm{v}=1)$ of NO have been studied, directly detecting not only $\mathrm{NO}^{+}$ions generated by autoionization but also every fragment atom produced by predissociation. In addition, the $\mathrm{v}=0$ states lying below the ionization threshold have also been investigated with the same method, and the comparison between the two results has more clearly elucidated the competing behavior of the decay processes in the above-threshold states $(v=1)$. As a result, for the $\mathrm{v}=1$ state, it has been shown that predissociation is the main decay process in the $\mathrm{np}$ Rydberg states, while autoionization is dominant in the ns Rydberg states. For the nf Rydberg states, the $\mathrm{N}\left({ }^{4} \mathrm{~S}\right)+\mathrm{O}\left({ }^{3} \mathrm{P}\right)$ predissociation channel, which has not been emphasized in previous studies, has been found to play an important role in the decay dynamics both above and below the ionization threshold.

KEY WORDS: autoionization, predissociation, resonance enhanced multiphoton ionization, Rydberg state, superexcited state, two-color double resonance excitation.

\section{INTRODUCTION}

Above the first ionization threshold, there are still many neutral states. These molecular states are called superexcited states, and have been considered to make important contribution to various chemical reactions. ${ }^{1,2}$ One example of such states is a high Rydberg state having a vibrationally excited ion core. In this type of states, which will be called a superexcited Rydberg state hereafter, one of the main decay processes is autoionization, in which vibrational energy of the ion core is converted to electronic energy. Another important decay process in highly excited states is predissociation, and the competition between these two processes is the central subject in the dynamics of superexcited states. In comparison with these main decay processes, radiative decay is generally much slower and almost negligible.

Although this autoionization-predissociation competition has extensively been studied so far by using synchrotron orbital radiation ${ }^{3-5}$ and laser multiphoton excitation 
techniques, ${ }^{6-9}$ its detailed feature has not yet been known. This may be partly because of the high state-density of superexcited states, which prevents us from clarifying the state-dependence of the competition. Another reason may be a difficulty in the observation of predissociation processes. The conventional method to find a predissociation decay is to observe a fluorescence decrease or linewidth broadening. However, we cannot apply this method to superexcited states, because autoionization also induces both effects. In addition, a superexcited state has generally several dissociation channels, and it is very difficult to distinguish each process with the conventional methods. The best way for detailed investigation of predissociation competing with autoionization is direct detection of dissociation fragments. ${ }^{10}$

In this work, we investigated the decay dynamics of high Rydberg states around the first ionization threshold of NO with a laser spectroscopic method. We used a two-color double resonance technique to achieve rotational-state-selective excitation of superexcited Rydberg states $(\mathrm{n}=8-12, l=\mathrm{s}$, $\mathrm{p}$, and $\mathrm{f}, \mathrm{v}=1)$, which lie above the first ionization threshold because of vibrational excitation. Both nitrogen and oxygen atoms produced by predissociation of a single rotational level of a superexcited Rydberg state were detected by using a resonance enhanced multiphoton ionization (REMPI) technique. Separate observation of three different spectra (absorption, fragment-atom-yield, and autoionization spectra) allowed us to study the competition between autoionization and predissociation in detail. The competition between vibrational autoionization and predissociation showed strong dependence on the orbital angular momentum and principal quantum number.

In addition to these measurements on superexcited Rydberg states, we observed decay processes of the $v=0$ levels as well. These levels lie below the first ionization threshold, and their autoionization channels are closed. The comparison between dynamical behaviors of the $\mathrm{v}=0$ and 1 levels allowed us more clearly to understand the complicated dynamical behavior in the superexcited states.

\section{EXPERIMENTAL}

Details of the experiment were described elsewhere. ${ }^{10}$ Typical excitation and probing schemes used in the experiment are shown in Figure 1. A pulsed laser pumps the NO molecule to the $\mathrm{N}=0, \mathrm{v}=0$ or 1 level of the $\mathrm{A}^{2} \Sigma^{+}(3 \mathrm{~s} \sigma)$ state (where $\mathrm{N}$ is a total angular momentum excluding electronic spin), and the second pulsed laser excites the molecule successively to high Rydberg states. In this excitation scheme, the Franck-Condon principle exclusively allows $\Delta v=0$ transitions because of the similarity between the potential curves for the A state and the Rydberg states. Therefore, vibrational levels of high Rydberg states are automatically selected by the vibrational selection in the intermediate A state. In the energy region around the final states, three dissociation channels are open, as shown in Figure 1, and atomic species producible by the predissociation are $\mathrm{N}\left({ }^{2} \mathrm{D}\right), \mathrm{N}\left({ }^{4} \mathrm{~S}\right), \mathrm{O}\left({ }^{1} \mathrm{D}\right)$, and $\mathrm{O}\left({ }^{3} \mathrm{P}\right)$. Each 

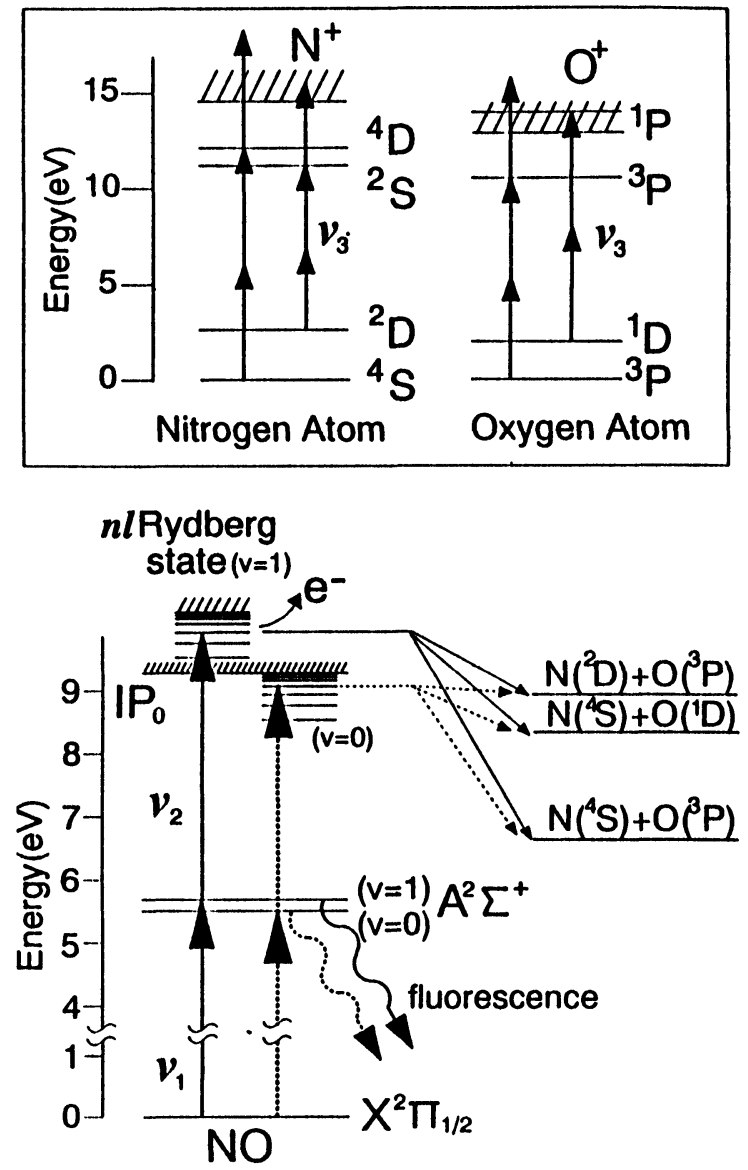

Figure 1 Excitation scheme for the NO molecule and probing schemes for the fragment atoms.

species is detected by REMPI with the third pulsed laser. There is no time delay between the two excitation lasers, but the third laser is delayed by $20 \mathrm{~ns}$ with respect to the excitation lasers.

In order to investigate the relative excitation efficiency of each Rydberg state, absorption intensity of the $\mathrm{n} l \leftarrow \mathrm{A}$ transition was also measured by the fluorescence dip method, ${ }^{11,12}$ in which the fluorescence intensity from the intermediate A state was detected to measure the population depletion caused by absorption of the second laser.

To eliminate collisional effects, we used a supersonic free jet expansion of pure $\mathrm{NO}$ gas. The $\mathrm{N}^{+}, \mathrm{O}^{+}$and $\mathrm{NO}^{+}$ions were separately detected with a time-of-flight mass-spectrometer. For extraction of ions to the flight tube, a pulsed electric field was applied to the interaction region so as to avoid field effects. 


\section{RESULTS AND DISCUSSION}

\subsection{Decay dynamics of superexcited Rydberg states $(v=1)$ : Competition} between autoionization and predissociation above the first ionization threshold

Figure 2 shows typical examples of the observed spectra, in which the first laser excited the molecule to the $\mathrm{v}=1, \mathrm{~N}=0$ level of the $\mathrm{A}^{2} \Sigma^{+}$state and the second laser wavelength was scanned around the $n l(v=1) \leftarrow \mathrm{A}^{2} \Sigma^{+}(\mathrm{v}=1)$ transition. In Figure

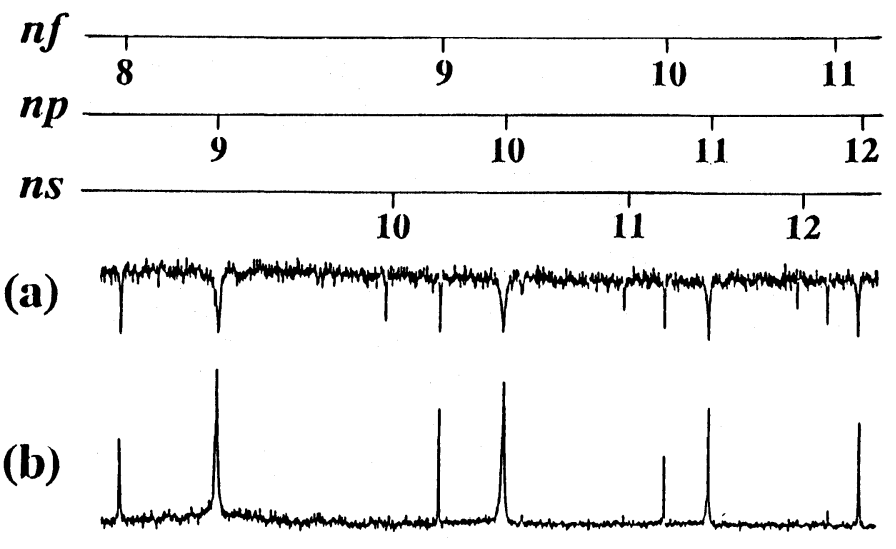

(c)

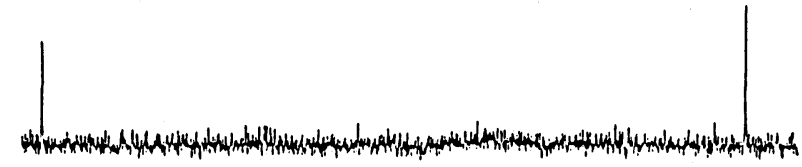

(d)

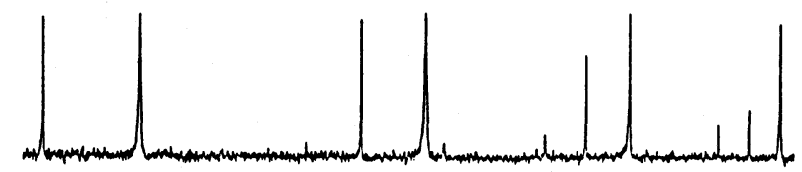

(e)

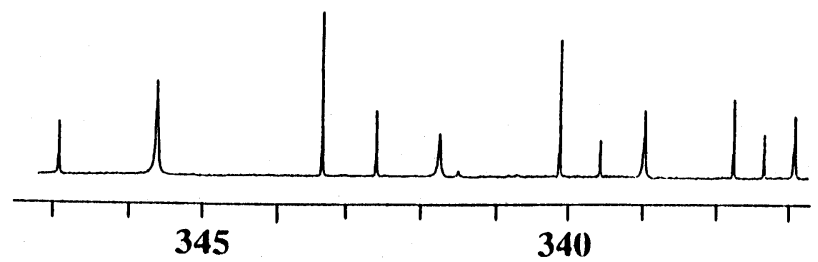

WAVELENGTH (nm)

Figure 2 Excitation spectra of NO observed when the second excitation laser wavelength is scanned around the $\mathrm{nl}(\mathrm{v}=1) \leftarrow \mathrm{A}^{2} \Sigma^{+}(\mathrm{v}=1)$ transition with the first excitation laser wavelength fixed at the $\mathrm{A}^{2} \Sigma^{+}(\mathrm{v}=1, \mathrm{~N}=0) \leftarrow \mathrm{X}^{2} \Pi$ transition; (a) fluorescence dip spectrum (fluorescence intensity from the intermediate A state), (b) $\mathrm{N}\left({ }^{2} \mathrm{D}\right)$-yield spectrum $\left(\mathrm{N}^{+}\right.$intensity detected with the probe laser fixed at the ${ }^{2} \mathrm{~S}_{1 / 2} \leftarrow{ }^{2} \mathrm{D}_{5 / 2}$ two-photon transition of the nitrogen atom), (c) $\mathrm{N}\left({ }^{4} \mathrm{~S}\right)$-yield spectrum (the same as (b) except that the probe laser is fixed at the ${ }^{4} \mathrm{D}_{7 / 2} \leftarrow{ }^{4} \mathrm{~S}_{3 / 2}$ ), (d) $\mathrm{O}\left({ }^{3} \mathrm{P}\right.$ )-yield spectrum $\left(\mathrm{O}^{+}\right.$intensity detected with the probe laser wavelength fixed at the ${ }^{3} \mathrm{P}_{\mathrm{J}} \leftarrow{ }^{3} \mathrm{P}_{2}$ two-photon transition of the oxygen atom), and (e) autoionization spectrum $\left(\mathrm{NO}^{+}\right.$intensity). 
2, the following spectra are shown: (a) fluorescence dip spectrum (in which the fluorescence intensity from the intermediate A state was monitored), (b) N( ${ }^{2} \mathrm{D}$ )-yield spectrum (in which $\mathrm{N}^{+}$ion intensity was measured when the third laser wavelength was fixed at the $3 p^{2} S_{1 / 2} \leftarrow 2 p^{3}{ }^{2} D_{5 / 2}$ two-photon transition of the nitrogen atom ${ }^{13}$ ), (c) $\mathrm{N}\left({ }^{4} \mathrm{~S}\right.$ )-yield spectrum (the same as (b) except that the third laser was fixed at the $3 p{ }^{4} D_{7 / 2} \leftarrow 2 p^{3}{ }^{4} S_{3 / 2}$ two-photon transition ${ }^{14,15}$ ), (d) $\mathrm{O}\left({ }^{3} \mathrm{P}\right.$ )-yield spectrum (in which $\mathrm{O}^{+}$ion intensity was measured when the third laser wavelength was fixed at the $3 p$ ${ }^{3} \mathrm{P}_{\mathrm{J}} \leftarrow 2 \mathrm{p}^{4}{ }^{3} \mathrm{P}_{2}$ two-photon transition of the oxygen atom ${ }^{14}$ ), and (e) autoionization spectrum $\left(\mathrm{NO}^{+}\right.$intensity). Although $\mathrm{O}\left({ }^{1} \mathrm{D}\right)$ was also attempted to detect, ${ }^{16}$ no signal was observed. Fine structure dependence ${ }^{17}$ of $N\left({ }^{2} \mathrm{D}_{\mathrm{J}}\right)$ and $\mathrm{O}\left({ }^{3} \mathrm{P}_{\mathrm{J}}\right)$ production was neglected in this study.

The peaks (dips) in the spectra consist of three Rydberg series, ns, $\mathrm{np}$, and $\mathrm{nf}$ $(\mathrm{n}=8-12, \mathrm{v}=1)$. Each peak corresponds to a single rotational level of a Rydberg state. The rotational levels of the $\mathrm{ns}, \mathrm{np}$, and $\mathrm{nf}$ Rydberg states are assigned to the $N=1(R=1), N=1(R=0)$, and $N=1(R=2)$ levels, respectively, where $R$ is a total angular momentum of the ion-core. These assignments were determined following previous spectroscopic studies by Anezaki et al. ${ }^{12}$ Their studies also show that the coupling between the orbital angular momentum of the Rydberg electron and the internuclear axis is so weak in these Rydberg states that they form " $l$ complex", in which various $\lambda$ components are mixed each other.

All the high Rydberg states seen in the spectra lie above the first ionization threshold due to their vibrational excitation, and they decay through vibrational autoionization, as shown in the spectrum (e). On the other hand, the three fragment-atom-yield spectra (b)-(d)) clearly indicate the presence of predissociation processes competing with the autoionization. The fact that the intensity distribution differs from spectrum to spectrum means that the decay processes of the superexcited nl Rydberg states strongly depend on their orbital angular momenta and principal quantum numbers.

The orbital angular momentum dependence of the decay dynamics is rather easy to understand. The $\mathrm{np}$ series shows the strongest absorption intensity, because the intermediate A state is the $3 \mathrm{~s} \sigma$ Rydberg state. While the $\mathrm{np}$ series is strong in the $\mathrm{N}\left({ }^{2} \mathrm{D}\right)$ - and $\mathrm{O}\left({ }^{3} \mathrm{P}\right)$-yield spectra, it is much weaker in the autoionization spectrum. These results are consistent with several previous experimental ${ }^{7,12,18,19}$ and theoreti$\mathrm{cal}^{20,21}$ studies, which predict that the predissociation to the $\mathrm{N}\left({ }^{2} \mathrm{D}\right)+\mathrm{O}\left({ }^{3} \mathrm{P}\right)$ limit is the main decay process in the $\mathrm{np}$ Rydberg states, and that the autoionization makes only minor contribution. Dissociative states for the predissociation of the np Rydberg states are considered to be the $\mathrm{B}^{2} \Pi$ and $\mathrm{L}^{2} \Pi$ states converging to the $\mathrm{N}\left({ }^{2} \mathrm{D}\right)+\mathrm{O}\left({ }^{3} \mathrm{P}\right)$ limit. Their potential curves 22 are shown in Figure 3.

In contrast to the $\mathrm{np}$ series, the ns series is strongest in the autoionization spectrum, while it is weakest in the absorption spectrum and shows only slight intensity in the fragment-atom-yield spectra. This suggests that autoionization is the dominant decay process in the ns Rydberg states. The presence of an interaction between the ns Rydberg states and $\mathrm{I}^{2} \Sigma^{+}$state converging to the $\mathrm{N}\left({ }^{2} \mathrm{D}\right)+\mathrm{O}\left({ }^{3} \mathrm{P}\right)$ limit has been indicated by a recent resonance multiphoton ionization-photoelectron measurement. ${ }^{6,23}$ However, it has also been suggested that this interaction is far weaker than that between the np and $B^{2} \Pi$ state. The present result supports this assertion. 


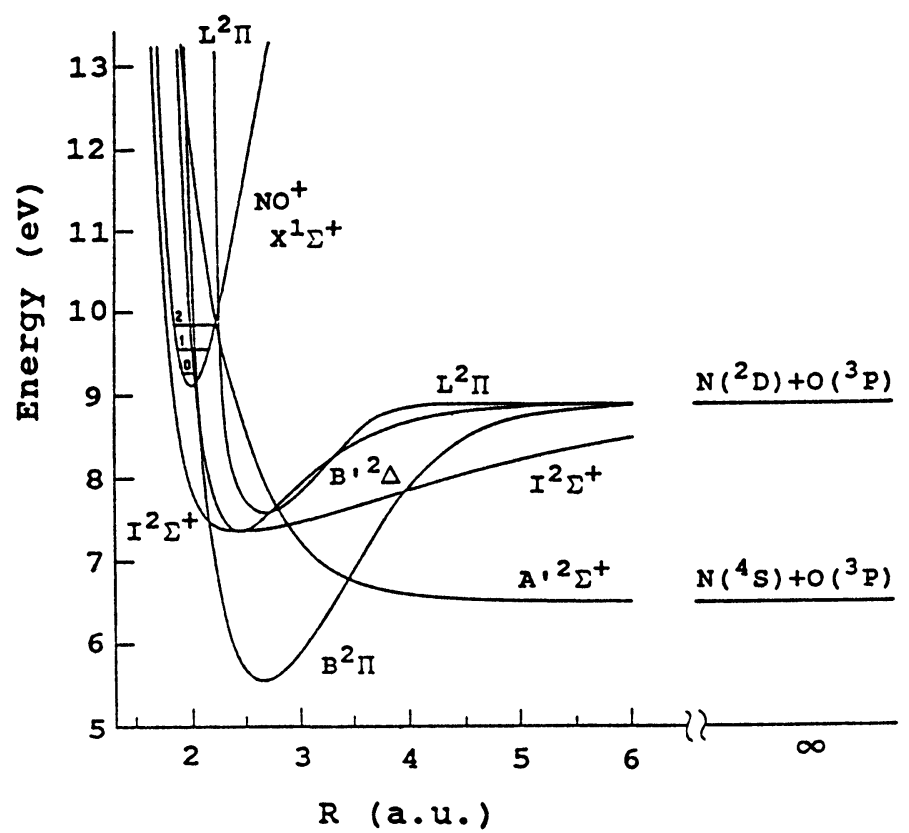

Figure 3 Potential curves of NO (from Ref. 22) associated with the present work. The curves of high Rydberg states are very close to that of the ground $\mathrm{X}^{1} \Sigma^{+}$state of $\mathrm{NO}^{+}$

The vibrational autoionization width $\left(\mathrm{cm}^{-1}\right)$ of $\mathrm{n} l \lambda$ Rydberg states is approximately given as, ${ }^{24-26}$

$$
\Gamma_{\text {vib }}=\frac{\mathrm{R \hbar}}{\mathrm{c}} \frac{\left(\delta_{(1)}^{\lambda}\right)^{2}}{\left(\mathrm{n}-\delta_{(0)}^{\lambda}\right)^{3}} \frac{\mathrm{v}}{\mathrm{m} \omega},
$$

where $\mathrm{R}$ is the Rydberg constant $\left(109735.3 \mathrm{~cm}^{-1}\right), \delta_{(0)}^{\lambda}$ is an adiabatic quantum defect at the equilibrium internuclear distance of the ion core, $\delta_{(1)}^{\lambda}$ is its first derivative with respect to the internuclear distance, $\mathrm{m}$ is the reduced mass, and $\omega$ is the harmonic vibrational frequency $\left(2376.4 \mathrm{~cm}^{-1}\right)$. The adiabatic quantum defects for $\mathrm{s} \sigma, \mathrm{p} \sigma$, and p $\pi$ Rydberg states of NO were calculated by Rault ${ }^{21}$ and Nakashima et al..$^{23}$ The vibrational autoionization widths $\left(\mathrm{cm}^{-1}\right)$ and corresponding rates $\left(\mathrm{s}^{-1}\right)$ for the $\mathrm{n}=10$ Rydberg states calculated from Eq.(1) are listed in Table 1. As stated above, the highly excited $p$ Rydberg states form "p-complex", and they are admixtures of $p \sigma$ and $\mathrm{p} \pi$ states. Diagonalizing a rotational Hamiltonian, ${ }^{12}$ it is shown that the observed rovibrational level of the $10 \mathrm{p}$ Rydberg state is a mixture of $8 \% \mathrm{p} \sigma$ and $92 \% \mathrm{p} \pi$ components. Therefore, the vibrational autoionization rate of this level is estimated to be $5.7 \times 10^{9}\left(\mathrm{~s}^{-1}\right)$. This value is about four times larger than that of the $10 \mathrm{~s} \sigma$ state. On the other hand, the present measurements show that the autoionization efficiency of the $10 \mathrm{~s}$ state is about five times larger than that of the $10 \mathrm{p}$ state. However, these results do not contradict each other, because the efficiency of a decay 
process does not depend on its own decay rate, but depends on a ratio among the rates of competing processes. The present results on the $p$ Rydberg states clearly indicate that the presence of the predissociation, whose rate is much larger than that of autoionization, greatly reduces the autoionization efficiency. The observed linewidth of the 10p Rydberg state is $3 \mathrm{~cm}^{-1}$, and this value is about hundred times broader than that expected from pure vibrational autoionization. This fact also proves the predominance of predissociation in the $\mathrm{np}$ states.

Table 1 Vibrational autoionization widths and rates of Rydberg states of NO

\begin{tabular}{lllll}
\hline$n l \lambda$ & $\delta^{\lambda}{ }_{(0)}{ }^{(a)}$ & $\delta^{\lambda}{ }_{(1)} / \mathrm{cm}^{-1(b)}$ & $\Gamma_{v i b} / \mathrm{cm}^{-1}$ & $k_{v i b} / s^{-1}$ \\
\hline $10 \mathrm{~s} \sigma$ & 1.12 & $6.6 \times 10^{6}$ & $8.1 \times 10^{-3}$ & $1.5 \times 10^{9}$ \\
$10 \mathrm{p} \sigma$ & 0.64 & $2.3 \times 10^{7}$ & $8.1 \times 10^{-2}$ & $1.5 \times 10^{10}$ \\
$10 \mathrm{p} \pi$ & 0.73 & $-1.2 \times 10^{7}$ & $2.6 \times 10^{-2}$ & $4.9 \times 10^{9}$ \\
\hline
\end{tabular}

(a) From Ref. 21-23

(b) From Ref. 21-23

The autoionization mechanism of the vibrationally excited np states has been investigated with resonance enhanced multiphoton ionization-photoelectron spectroscopy by Kimman et al. ${ }^{7}$ They showed that vibrational distribution of product ions strongly deviates from a propensity rule for vibrational autoionization $(\Delta v=-1)$. Therefore, we can infer that not only vibrational autoionization but also electronic autoionization via dissociative valence states largely contribute to the autoionization decay in the np Rydberg states.

Regarding the predissociation in the nf. Rydberg states, there have been few reports except for the lowest $4 \mathrm{f}$ state. ${ }^{27,28}$ This may be reasonable because the predissociation in $\mathrm{f}$ Rydberg states has generally been expected to be very weak because of a high centrifugal barrier, which prevents the $f$ Rydberg electron from deep penetration into the ion core. However, in contrast to this traditional understanding, the present spectra provide interesting results: we can see from Figure 2 that $N\left({ }^{2} D\right)$ and $\mathrm{O}\left({ }^{3} \mathrm{P}\right)$ production efficiencies of the nf states are close to those of the strongly predissociating $\mathrm{np}$ states. This means that predissociation plays a very important role in the nf states as well, and is quite contrast to previous predictions and somewhat surprising at first sight. However, this is not unreasonable because the small interaction between the ion core and Rydberg electron also makes the autoionization rate slow. It should be noted again that the efficiency of each process is determined by a ratio among the rates of all processes. There has been no information on the coupling between the nf and valence states converging to the $N\left({ }^{2} \mathrm{D}\right)+\mathrm{O}\left({ }^{3} \mathrm{D}\right)$ limit. Therefore, the dissociative states responsible for this $\mathrm{nf}$ predissociation is difficult to determine at present.

Another interesting result on the $\mathrm{f}$ Rydberg states is the production of $\mathrm{N}\left({ }^{4} \mathrm{~S}\right)$ atoms. There have been neither theoretical predictions nor experimental reports on the $N\left({ }^{4} S\right)$ production from high Rydberg states of NO. Our result is the first evidence of $\mathrm{N}\left({ }^{4} \mathrm{~S}\right)$ generating predissociation, and clearly demonstrates the importance of direct observation of predissociation processes. The $\mathrm{N}\left({ }^{4} \mathrm{~S}\right)$-yield spectrum is characterized 
by strong $\mathrm{n}$-dependence. This strong state-dependence will be discussed in the next subsection.

There are two open dissociation channels that can produce the $\mathrm{N}\left({ }^{4} \mathrm{~S}\right)$ atoms. They are the $\mathrm{N}\left({ }^{4} \mathrm{~S}\right)+\mathrm{O}\left({ }^{1} \mathrm{D}\right)$ and $\mathrm{N}\left({ }^{4} \mathrm{~S}\right)+\mathrm{O}\left({ }^{3} \mathrm{P}\right)$ channels. All molecular states derived from the $\mathrm{N}\left({ }^{4} \mathrm{~S}\right)+\mathrm{O}\left({ }^{1} \mathrm{D}\right)$ combination have quartet spin multiplicity, and their interaction with the doublet Rydberg states is expected to be small. Therefore, it is reasonable to attribute the observed $\mathrm{N}\left({ }^{4} \mathrm{~S}\right)$ production to dissociative states converging to the $\mathrm{N}\left({ }^{4} \mathrm{~S}\right)+\mathrm{O}\left({ }^{3} \mathrm{P}\right)$ limit. This is supported by the present experimental results that no $\mathrm{O}\left({ }^{\mathrm{D}} \mathrm{D}\right)$ signal was observed, and that the $\mathrm{O}\left({ }^{3} \mathrm{P}\right)$ production efficiency is particularly large in the $8 \mathrm{f}$ and $11 \mathrm{f}$ Rydberg states, which efficiently produce $\mathrm{N}\left({ }^{4} \mathrm{~S}\right)$ atoms as well. Only two doublet states, $\mathrm{X}^{2} \Pi$ and $\mathrm{A}^{\prime 2} \Sigma^{+}$, can be derived from the $\mathrm{N}\left({ }^{4} \mathrm{~S}\right)+\mathrm{O}\left({ }^{3} \mathrm{P}\right)$ combination. ${ }^{29}$ The potential curve of the ground $\mathrm{X}^{2} \Pi$ state is much separated from those of the Rydberg states, so we can conclude that the observed $N\left({ }^{4} S\right)$ production is caused by the $\mathrm{A}^{\prime 2} \Sigma^{+}$state. The contribution of the $\mathrm{A}^{\prime 2} \Sigma^{+}$state was also proved by rotational state selectivity of the $\mathrm{N}\left({ }^{4} \mathrm{~S}\right)$ production in the $7 \mathrm{f}$ state $(\mathrm{v}=1)$ in our previous work. ${ }^{10}$

\subsection{Decay dynamics of high Rydberg states $(v=0)$ below the first ionization threshold}

When we excite the $v=0$ level of the intermediate A state, only $v=0$ levels of high Rydberg states axe excited in the second excitation process because the FranckCondon principle allows $\Delta v=0$ transitions exclusively. Figure 4 shows typical examples of the observed spectra, in which the first laser excited the molecule to the $v=0, N=0$ level of the $A^{2} \Sigma^{+}$state and the second laser wavelength was scanned around the $\mathrm{nl}(\mathrm{v}=0) \leftarrow \mathrm{A}(\mathrm{v}=0)$ transition. In Figure 4, the following spectra are shown: (a) fluorescence dip (absorption) spectrum, (b) N( ${ }^{2} \mathrm{D}$ )-yield spectrum, and (c) $\mathrm{N}\left({ }^{4} \mathrm{~S}\right)$-yield spectrum. These spectra were measured by the same methods as in Figure 2.

The observed $\mathrm{v}=0$ levels of high Rydberg states lie below the first ionization threshold, and their autoionization channels are, of course, closed. In addition, direct ionization cross sections of these states are negligibly small. Therefore, an ionization spectrum (in which $\mathrm{NO}^{+}$intensity is monitored) in this energy region shows no Rydberg structure. All the Rydberg peaks in the fluorescence dip spectrum appear in either the $\mathrm{N}\left({ }^{2} \mathrm{D}\right)$ - or $\mathrm{N}\left({ }^{4} \mathrm{~S}\right)$-yield spectrum. For example, while the intensities of the $8 \mathrm{f}$ and $10 \mathrm{f}$ states are explicitly small in the $\mathrm{N}\left({ }^{2} \mathrm{D}\right)$-yield spectrum, they are strong in the $\mathrm{N}\left({ }^{4} \mathrm{~S}\right)$-yield, compensating the weakness in the former spectrum. These facts show that the decay processes of these Rydberg states are characterized by the competition between the two predissociation processes, $\mathrm{N}\left({ }^{2} \mathrm{D}\right)+\mathrm{O}\left({ }^{3} \mathrm{P}\right)$ and $\mathrm{N}\left({ }^{4} \mathrm{~S}\right)+\mathrm{O}\left({ }^{3} \mathrm{P}\right)$, as described in the previous subsection.

To clarify the difference between the $\mathrm{v}=0$ and $\mathrm{v}=1$ levels, we present Figures 5-7, in which the spectra observed in these two different levels are shown together. Figure 5 shows the fluorescence dip spectra. The horizontal axis of each spectrum is the wavelength of the second excitation laser. Both $\mathrm{v}=0$ and 1 levels appear at almost the same wavelength because of the similarity between the vibrational poten- 


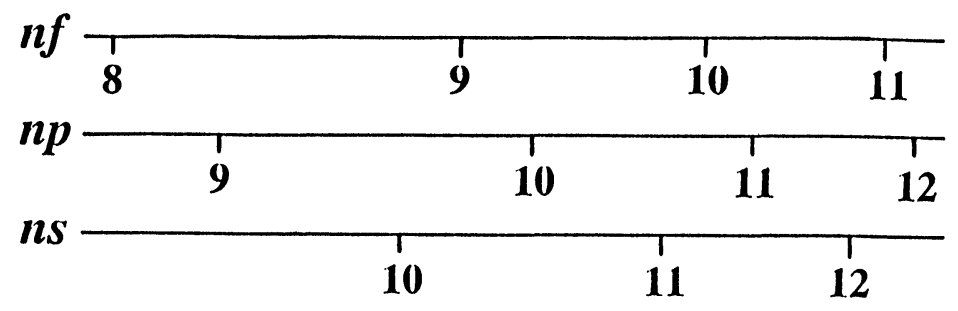

(a)
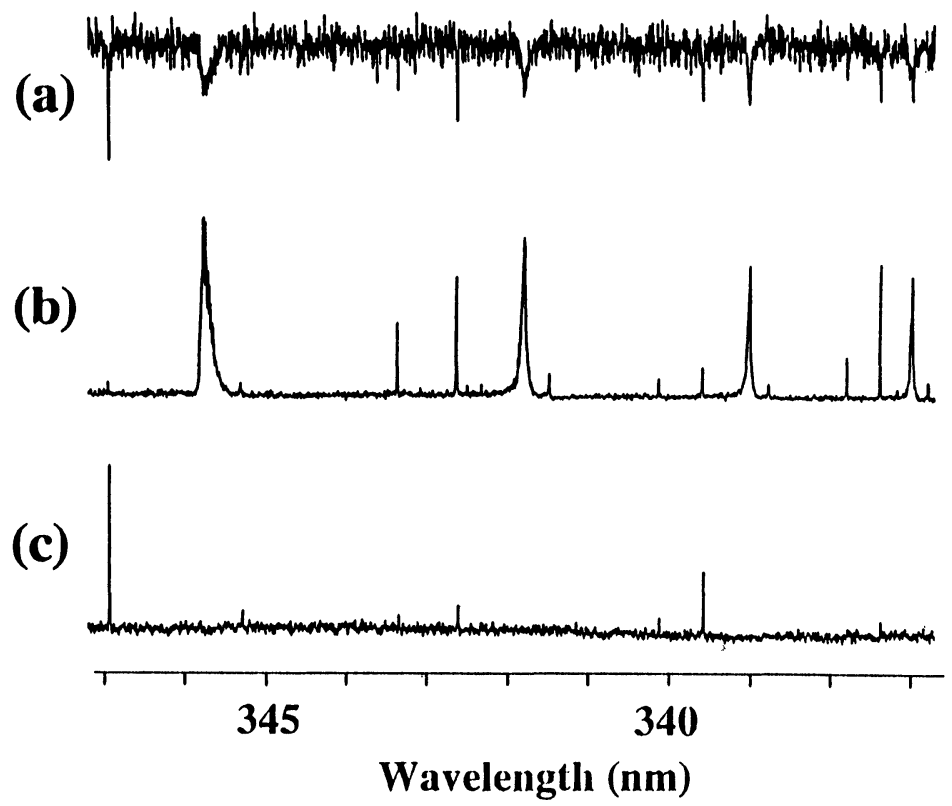

Figure 4 Excitation spectra of NO observed in the same way as those in Figure 2 except that the first excitation laser wavelength fixed at the transition to the $\mathrm{v}=0, \mathrm{~N}=0$ level of the $\mathrm{A}^{2} \Sigma^{+}$state and the second excitation laser wavelength is scanned around the $\mathrm{nl}(\mathrm{v}=0) \leftarrow \mathrm{A}^{2} \Sigma^{+}(\mathrm{v}=0)$ transitions; (a) fluorescence dip spectrum, (b) $\mathrm{N}\left({ }^{2} \mathrm{D}\right)$-yield spectrum, (c) $\mathrm{N}\left({ }^{4} \mathrm{~S}\right)$-yield spectrum.

tials for the intermediate A state and the high Rydberg states. Intensity distributions of these two spectra are very similar to each other. This is reasonable because the electronic and rotational parts of transition moments are common and the vibrational parts (Franck-Condon factors) are diagonalized.

Figure 6 allows us to compare the behaviors of the $\mathrm{v}=0$ and $\mathrm{v}=1$ levels in the $\mathrm{N}\left({ }^{2} \mathrm{D}\right)$-yield spectra. The $\mathrm{np}$ series are strong in both levels, and there is no explicit difference between the intensity distributions of the np series. The linewidths of the $\mathrm{v}=0$ levels of the $10 \mathrm{p}, 11 \mathrm{p}$, and $12 \mathrm{p}$ states are 6,3 , and $3 \mathrm{~cm}^{-1}$, respectively. Therefore, it is obvious that the radiative decay process is completely negligible in the $\mathrm{np}$ Rydberg states, since the radiative lifetimes of these states are estimated to be hundreds ns. In addition, the transition to the $v=0$ level of the $9 p$ state shows an asymmetric lineshape. This is considered to be the Fano shape caused by interference with a transition to a dissociative continuum. ${ }^{25,30}$ Only a few observations of 


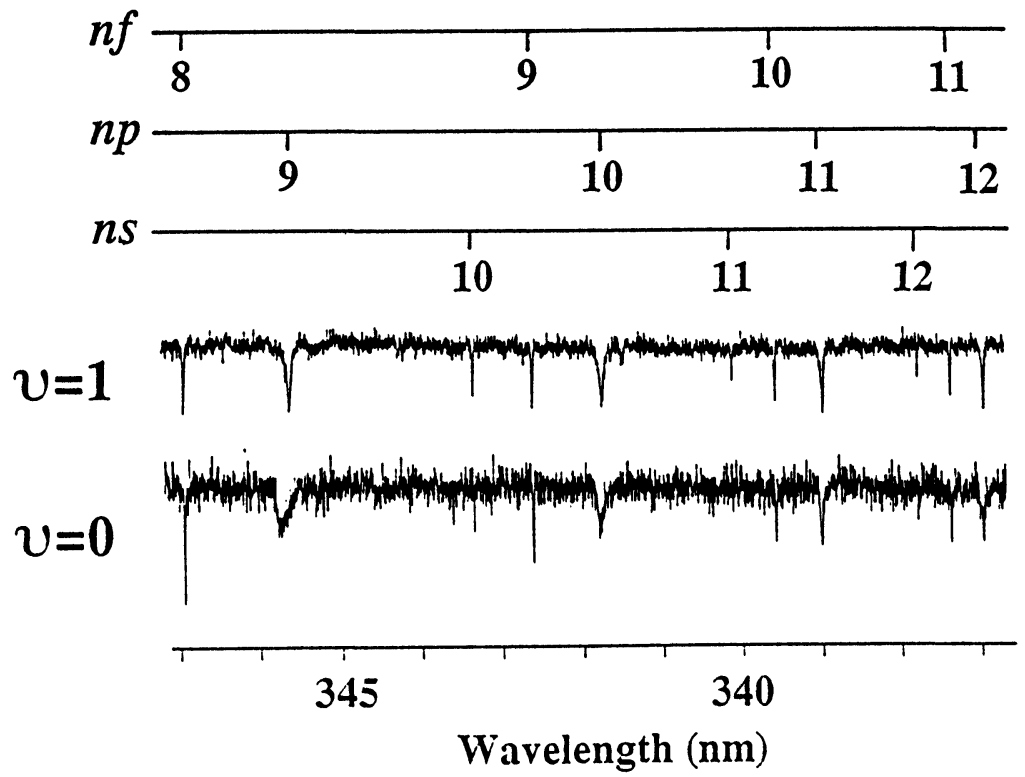

Figure 5 Comparison of the fluorescence dip (= absorption) spectrum of the $\mathrm{v}=0$ level with the corresponding spectrum of the $\mathrm{v}=1$ level.

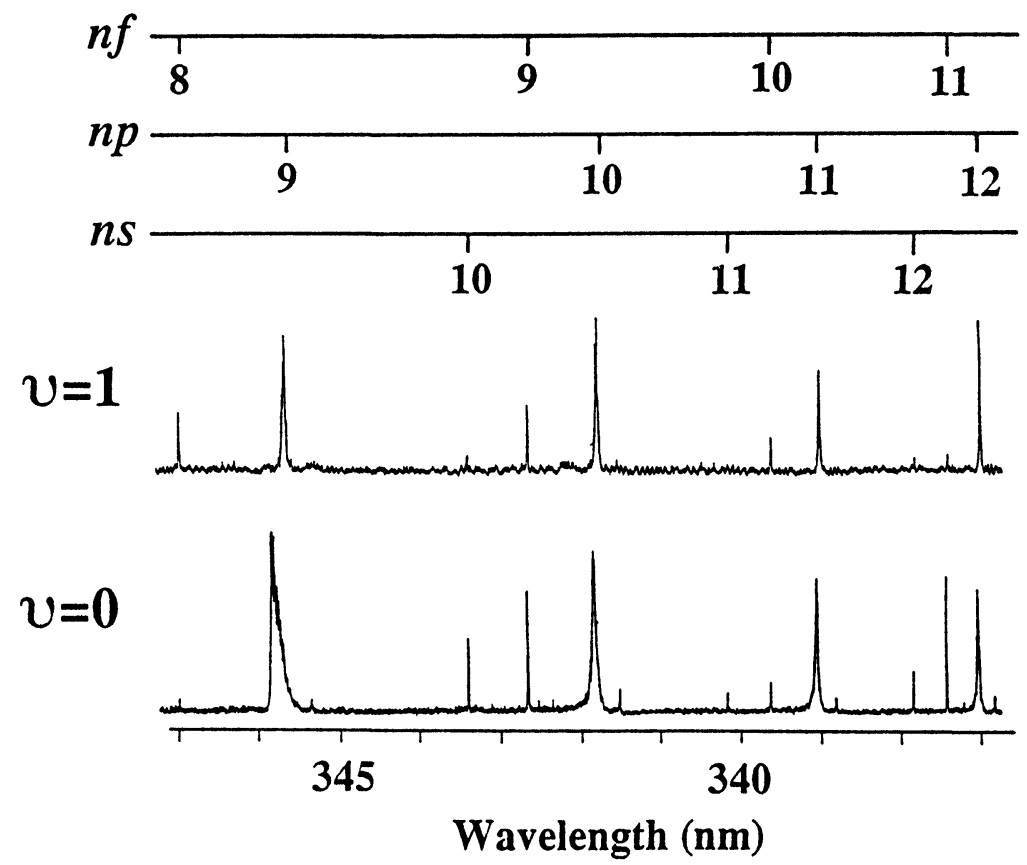

Figure 6 Comparison of the $N\left({ }^{2} \mathrm{D}\right)$-yield spectrum of the $\mathrm{v}=0$ level with the corresponding spectrum of the $\mathrm{v}=1$ level. 


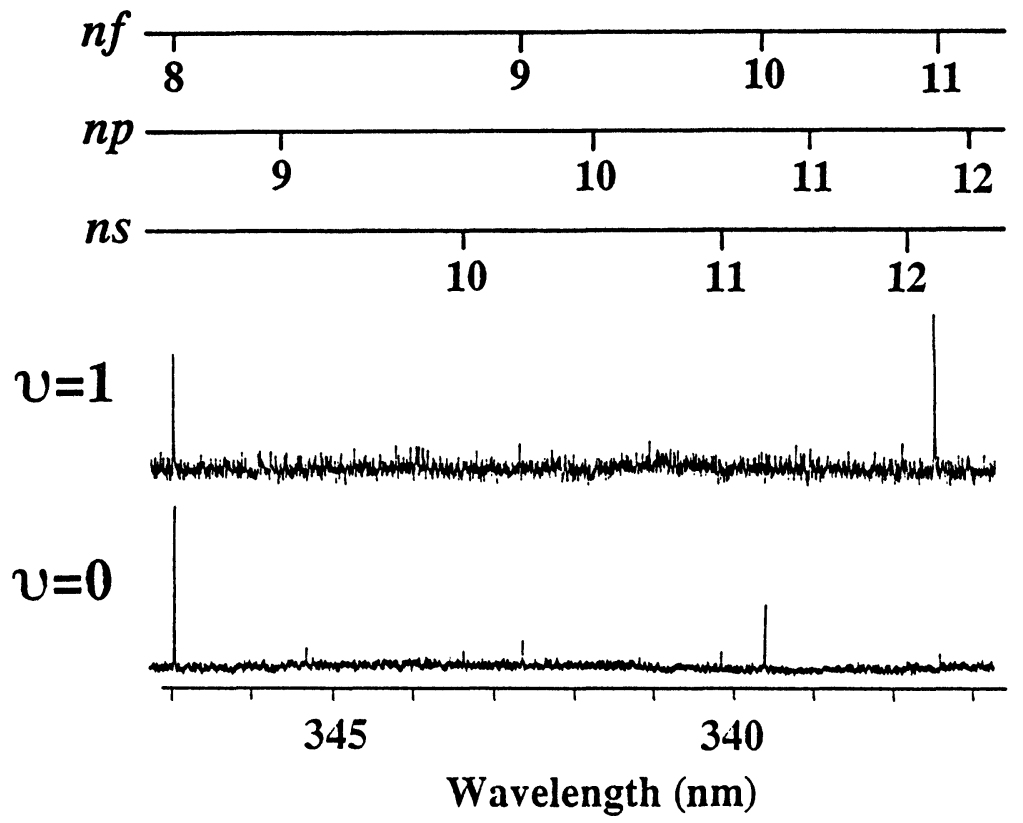

Figure 7 Comparison of the $\mathrm{N}\left({ }^{4} \mathrm{~S}\right)$-yield spectrum of the $\mathrm{v}=0$ level with the corresponding spectrum of the $\mathrm{v}=1$ level.

the Fano shape due to dissociative continuum have been reported so far, while there have been many observations of those due to ionization continuum. It is interesting that only the $9 p$ state shows the remarkable Fano shape, though its reason is not clear at present.

The most remarkable difference between the two N($\left.{ }^{2} \mathrm{D}\right)$-yield spectra in Figure 6 lies in the intensity of the ns series. While the ns series is very weak in the $\mathrm{v}=1$ spectrum, it is much stronger in $v=0$. This result indicates that the $N\left({ }^{2} \mathrm{D}\right)$ production efficiencies are much enhanced in the $v=0$ level compared with those of the $v=$ 1 level, because the absorption intensities of the ns series are almost the same in these two vibrational levels, as seen in Figure 5. This enhancement of the predissociation efficiency is caused by the lack of autoionization channels in the $\mathrm{v}=0$ level, and shows the importance of the autoionization in the decay dynamics of the superexcited ns Rydberg states. In addition, Figure 4 shows that the intensity ratio between the $n p$ and ns Rydberg series in the $N\left({ }^{2} \mathrm{D}\right)$-yield spectrum is roughly the same as in the fluorescence dip spectrum. This result indicates that also in the ns Rydberg states the predissociation to $\left.\mathrm{N}^{2} \mathrm{D}\right)+\mathrm{O}\left({ }^{3} \mathrm{P}\right)$ is the main decay process in the levels below the ionization threshold, and that the radiative process makes only minor contribution.

In the observed energy region, the decay dynamics of the nf Rydberg states show remarkable dependence on the principal quantum number, while those of the ns and $\mathrm{np}$ Rydberg states do not strongly depend. Figure 7 , which compares the $\mathbf{N}\left({ }^{4} \mathrm{~S}\right)-$ yield spectra of the $\mathrm{v}=0$ and 1 levels, clearly demonstrates a complicated behavior 
of the decay dynamics in the nf Rydberg states. In the spectrum of the $\mathrm{v}=1$ level, the $8 \mathrm{f}$ and $11 \mathrm{f}$ are considerably strong, while the $9 \mathrm{f}$ and $10 \mathrm{f}$ are very weak. On the other hand, in the spectrum of the $v=0$ level, while the $11 \mathrm{f}$ is not seen, the intensity of the $10 \mathrm{f}$ states is remarkably enhanced. The nf states being absent in the $\mathrm{N}\left({ }^{4} \mathrm{~S}\right)$-yield spectrum show strong peaks in the $\mathrm{N}\left({ }^{2} \mathrm{D}\right)$-yield spectrum, as seen in Figure 2 and 4. For example, while the $11 \mathrm{f}$ states with $\mathrm{v}=0$ is completely absent in the $\mathrm{N}\left({ }^{4} \mathrm{~S}\right)$-yield spectrum, its intensity is quite strong in the $\mathrm{N}\left({ }^{2} \mathrm{D}\right)$-yield spectrum. These results clearly show that the competition between the two predissociation processes in the nf states strongly depends on both the principal quantum number and vibrational level. Though the state-dependence of the decay dynamics is so drastic, its simple explanation seems hardly possible. The Franck-Condon factors cannot explain such a strong vibrational dependence, because the potential curves of these states are very similar to each other and the energy separations among the curves are about $850 \mathrm{~cm}^{-1}$ at most. More probably, there may be complicated channel interactions, and this remarkable state-dependence may be one of the manifestations of those interactions.

\section{CONCLUSIONS}

In this work, we have investigated the state-dependence of decay dynamics in high Rydberg states of NO by laser spectroscopic methods. Not only autoionization decay but also two predissociation processes have been studied with direct detection of ions and fragment atoms. To our knowledge, this is the first experimental study to monitor all the possible decay channels of high Rydberg states around the ionization threshold. Above the ionization threshold, the competition between autoionization and predissociation has been found to show strong dependence on the orbital angular momentum and principal quantum number. The comparison with the spectra below the ionization threshold has given us a great help in understanding the significance of the autoionization process in the superexcited states. On the other hand, remarkable state-dependence of the competition between the two predissociation processes in the nf Rydberg states suggests the presence of complicated channel interactions. It is an important future study to investigate this problem.

\section{References}

1. H. Nakamura. Int. Rev. Phys. Chem., 10, 123 (1991).

2. Y. Hatano. in Dynamics of excited molecules., edited by K. Kuchitsu, (Elsevier, Amsterdam, 1993).

3. P. M. Guyon, T. Baer and I. Nenner. J. Chem. Phys., 78, 3665 (1983).

4. M. Ukai, K. Kameta, N. Kouchi, K. Nagano, Y. Hatano and K. Tanaka. J. Chem. Phys., 97, 2835 (1992).

5. S. B. Kim, D. J. Kane, and J. G. Eden. Phys. Rev. Lett., 68, 1311 (1992).

6. Y. Achiba, K. Sato and K. Kimura. J. Chem. Phys., 82, 3959 (1985); Y. Achiba and K. Kimura. Chem. Phys., 129, 11 (1989).

7. J. Kimman, J. W. J. Verschuur, M. Lavolle, H. B. van Linden van den Heuvell and M. J. van der Wiel. J. Phys., B 19, 3909 (1986). 
8. S. T. Pratt, J. L. Dhemer, and P. M. Dehmer. J. Chem. Phys., 90, 2201 (1989); S. T. Pratt, Ch. Jungen, and E. Miescher, ibid 90, 5971 (1989).

9. F. X. Campos, Y. J. Jiang, and E. R. Grant. J. Chem. Phys., 93, 7731 (1990).

10. A. Fujii and N. Morita. Chem. Phys. Lett., 182, 304 (1991); J. Chem. Phys., 97327 (1992); ibid 98, 4581 (1993).

11. T. Ebata, N. Mikami, and M. Ito. J. Chem. Phys., 78, 1132 (1983).

12. Y. Anezaki, T. Ebata, N. Mikami, and M. Ito. Chem. Phys., 89, 103 (1984); ibid 97, 153 (1985).

13. G. Black, and L. Jusinski. Chem. Phys. Lett., 139, 41 (1987).

14. W. K. Bischel, B. E. Perry, and D. R. Crosley. App. Opt., 21, 1419 (1982).

15. C. P. Fell, J. I. Steinfeld, and S. M. Miller. Spectrochim. Acta., 46A, 431 (1990).

16. S. T. Pratt, P. M. Dehmer, and J. L. Dehmer. Phys. Rev., A43, 282(1991).

17. Y. Matumi and M. Kawasaki. J. Chem. Phys., 93, 2481 (1990).

18. E. Miescher. Can. J. Phys., 54, 2074 (1976).

19. D. T. Biernacki, S. D. Colson, and E. E. Eyler. J. Chem. Phys., 88, 2099 (1988).

20. A. Giusti-Suzor and Ch. Jungen. J. Chem. Phys., 80, 986 (1984).

21. M. Rault. J. Chem. Phys., 87, 4736 (1987).

22. H. Sun and H. Nakamura. J. Chem. Phys., 93, 6491 (1990).

23. K. Nakashima, H. Nakamura, Y. Achiba, and K. Kimura. J. Chem. Phys., 91, 1603 (1989).

24. G. Herzberg and Ch. Jungen. J. Mol. Spectrosc., 41, 425 (1972).

25. H. Lefebvre-Brion and R. W. Field. Perturbations in the Spectra of Diatomic Molecules (Academic Press, Orland, 1986).

26. H. Takagi and H. Nakamura. J. Chem. Phys., 74, 5808 (1981).

27. Ch. Jungen and E. Miescher. Can. J. Phys., 47, 1769 (1969).

28. K. P. Huber, M. Vervloet, Ch. Jungen and A. L. Roche. Mol. Phys., 61, 501 (1987).

29. H. H. Michels. Adv. Chem. Phys., 45, 225 (1981).

30. U. Fano. Phys. Rev., 124, 1866 (1961). 